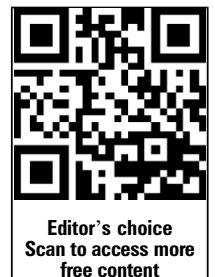

${ }^{1}$ Department of Clinical Neurophysiology, Danish Center for Sleep Medicine, Glostrup, Denmark

${ }^{2}$ Faculty of Health Sciences, Center for Healthy Aging, University of Copenhagen, Glostrup Hospital,

Copenhagen, Denmark 3 itracks, Aarhus, Denmark ${ }^{4}$ Danish Institute for Local and Regional Government Research, Copenhagen, Denmark

\section{Correspondence to} Professor Poul Jennum, Danish Centre for Sleep Medicine, Department of Clinical Neurophysiology, Faculty of Health Sciences, University of Copenhagen, Glostrup Hospital, Glostrup DK 2600, Denmark POJE@glo.regionh.dk

Received 12 August 2012 Revised 26 April 2013 Accepted 11 May 2013 Published Online First 8 June 2013

\title{
Morbidity and mortality in children with obstructive sleep apnoea: a controlled national study
}

\author{
Poul Jennum, ${ }^{1,2}$ Rikke Ibsen, ${ }^{3}$ Jakob Kjellberg ${ }^{4}$
}

\begin{abstract}
Background Little is known about the diagnostic patterns of obstructive sleep apnoea (OSA) in children. A study was undertaken to evaluate morbidity and mortality in childhood OSA.

Methods 2998 patients aged 0-19 years with a diagnosis of OSA were identified from the Danish National Patient Registry. For each patient we randomly selected four citizens matched for age, sex and socioeconomic status, thus providing 11974 controls. Results Patients with OSA had greater morbidity at least 3 years before their diagnosis. The most common contacts with the health system arose from infections (OR 1.19, 95\% Cl 1.01 to 1.40); endocrine, nutritional and metabolic diseases (OR 1.30, 95\% Cl 0.94 to 1.80); nervous conditions (OR 2.12,95\% Cl 1.65 to 2.73); eye conditions (OR 1.43, 95\% Cl 1.07 to 1.90); ear, nose and throat (ENT) diseases (OR 1.61, 95\% Cl 1.33 to 1.94); respiratory system diseases (OR $1.78,95 \% \mathrm{Cl} 1.60$ to 1.98); gastrointestinal diseases (OR $1.34,95 \% \mathrm{Cl} 1.09$

\section{Key messages}

What is the key question?

- What are the morbidities and mortalities in children with a diagnosis of obstructive sleep apnoea?

What is the bottom line?

- Children with obstructive sleep apnoea have significant morbidities before and after diagnosis, and their 5-year death rates are generally higher than those of control subjects.

\section{Why read on?}

- This study describes serious morbidity and increased mortality in children diagnosed with obstructive sleep apnoea. Closer attention should be paid to high-risk children.
\end{abstract} to 1.66$)$; skin conditions (OR $1.32,95 \% \mathrm{Cl} 1.02$ to 1.71); congenital malformations (OR $1.56,95 \% \mathrm{Cl} 1.31$ to 1.85); abnormal clinical or laboratory findings (OR $1.21,95 \% \mathrm{Cl} 1.06$ to 1.39$)$; and other factors influencing health status (OR 1.29, 95\% Cl 1.16 to 1.43). After diagnosis, OSA was associated with incidences of endocrine, nutritional and metabolic diseases (OR 1.78, $95 \% \mathrm{Cl} 1.29$ to 2.45 ), nervous conditions (OR 3.16, 95\% $\mathrm{Cl} 2.58$ to 3.89), ENT diseases (OR 1.45, 95\% Cl 1.14 to 1.84), respiratory system diseases (OR $1.94,95 \% \mathrm{Cl} 1.70$ to 2.22 ), skin conditions (OR $1.42,95 \% \mathrm{Cl} 1.06$ to 1.89), musculoskeletal diseases (OR $1.29,95 \% \mathrm{Cl} 1.01$ to 1.64 ), congenital malformations (OR $1.83,95 \%$ $\mathrm{Cl} 1.51$ to 2.22), abnormal clinical or laboratory findings (OR $1.16,95 \% \mathrm{Cl} 1.06$ to 1.27 ) and other factors influencing health status $(\mathrm{OR} 1.35,95 \% \mathrm{Cl} 1.20$ to 1.51). The 5 -year death rate was 70 per 10000 for patients and 11 per 10000 for controls. The HR for cases compared with controls was 6.58 (95\% Cl 3.39 to 12.79 ; $\mathrm{p}<0.001)$

Conclusions Children with OSA have significant morbidities several years before and after their diagnosis.

\section{INTRODUCTION}

Obstructive sleep apnoea (OSA) affects a significant proportion of the population. It increases the risk of cardiovascular and cerebrovascular morbidity and mortality in the adult population. Most studies have focused on adult OSA with respect to the deleterious effects on patients' social function, employment and quality of life. However, recent studies suggest that OSA is not benign in children either. It is relatively common in children, in whom it is associated with overweight, ${ }^{1}{ }^{2}$ craniofacial abnormalities, ${ }^{3}$ neurological ${ }^{4}$ and otolaryngeal diseases. OSA is associated with cardiovascular changes, including ECG and blood pressure (BP) changes, ${ }^{5}$ excessive daytime sleepiness, ${ }^{67}$ cognitive impairment,${ }^{8} 9$ behavioural changes, ${ }^{10}$ school and educational problems ${ }^{9}{ }^{11}$ and reduced quality of life. ${ }^{12}$ Although these issues were identified many years ago, OSA is still underdiagnosed in children. ${ }^{13}$ Furthermore, there are almost no studies comparing morbidity before and after a diagnosis of OSA and mortality due to OSA in children with control child subjects.

In Denmark it is possible to evaluate the general disease pattern of all patients with specific diagnoses from hospitals and clinics in the primary and secondary care sectors by interrogating a central database, the National Patient Registry (NPR). We used the NPR to evaluate morbidity before an OSA diagnosis. In a previous study we used the NPR to address the socioeconomic consequences of OSA. ${ }^{14}$ The aim of this study was to identify patients' health and diseases before and after a diagnosis of OSA.

\section{METHODS \\ Subjects}

In Denmark all hospital inpatient and outpatient contacts are obliged to be recorded in the NPR. The information supplied to the NPR includes administrative information (eg, time of contact) and information about all primary and secondary 
diagnoses and diagnostic procedures (eg, polysomnography) and treatment procedures (eg, surgery or continuous positive airway pressure, CPAP) using several international classification systems including the International Classification of Disorders (ICD-10). The thorough and accurate nature of the NPR means that the data extracted are representative of all patients in Denmark who have received a diagnosis of OSA, irrespective of any other diagnoses. The diagnosis of OSA was made on the basis of clinical practice irrespective of the methods used for diagnosis (polysomnography (PSG), polygraphy or oximetry). As data are available for the entire observation period, we can trace patients retrospectively and prospectively relative to the time of their diagnosis. Furthermore, all contacts in the primary sector (general practice and specialist care such as paediatricians or ear, nose and throat (ENT) specialists in the primary sector) and the use of any medication are recorded in the National Health Security and Danish Medicine Agency databases, respectively. There are limitations to the NPR: (1) a small fraction of patients who receive their diagnosis in the primary sector are not identified; (2) the control group may include unidentified OSA patients; (3) specific clinical information such as body mass index (BMI), apnoea-hypopnoea index and BP are not recorded sufficiently thoroughly in the NPR and therefore cannot be included in the analysis.

Using the NPR, we identified all patients who were diagnosed with OSA between 1997 and 2009. For the OSA diagnoses we used the International Classification of Diseases (ICD-9) in Denmark; these codes are given after patient evaluation in each hospital (based on PSG/polygraphy). Using data from Denmark's Civil Registration System Statistics, we then randomly selected citizens of the same age and gender who did not have a diagnosis of sleep-disordered breathing including snoring, OSA or hypoventilation. We attempted to match patients for socioeconomic status (SES) by selecting control subjects from the same locality (typically 30-100 000 inhabitants) where each patient lived. The ratio of control subjects to patients was 4:1 in order to reduce variation among controls. Data from patients and matched control subjects who could not be identified in the Coherent Social Statistics database were excluded from the sample. All observations in the two groups were successfully matched.

The patients and matched control subjects were followed from the year of diagnosis until 2009. We also controlled for parental education and income level. Adjusting for parental social factors may be relevant for children but causes problems when comparing with OSA in adults. In choosing a control group it is difficult to decide the extent to which morbidities should be excluded and whether to control for SES status. Certain diseases are associated with low SES (eg, obesity) or cause social deviation (eg, narcolepsy). We chose to use the same selection of controls in childhood as in adult OSA to facilitate their comparison. We noted a bimodal age distribution of patients with a minimum age of 20 in the NPR, so we decided to include patients aged $0-19$ years in the study.

\section{Evaluation of morbidity before a diagnosis of OSA}

Information from the period before OSA diagnosis was extracted from the database for the years 1997-2009. Morbidity data were extracted as primary and secondary diagnoses and subdivided into major disease groups in accordance with WHO ICD-10 criteria. ORs and 95\% CIs were calculated for the main diagnoses. We performed subgroup analyses of the major diagnostic groups which showed significant differences to identify the most common diagnosis in each category that was associated with a diagnosis of OSA. We only report all-cause mortality rates because these often refer to the obvious cause of death (eg, pneumonia), which tends to underestimate the underlying mechanism, and because the relatively small number of child deaths means that any subgroups would be too small to detect statistically significant differences of the magnitude we might reasonably expect. We used a limit of comorbid disease occurrence of more than $1 \%$ to select diseases, and then calculated the OR for each of them.

\section{Statistical analysis}

Data were analysed by conditional logit modelling using SAS V.9.1.3 (SAS, Cary, North Carolina, USA). The dependent variable was the binary variable for case-control groups and the independent variables were those of the 21 diagnosis groups, omitting the group with no diagnosis 3 years before the diagnosis. The second analysis included dummy variables for ICD-10 diagnoses in which the incidence was $>1 \%$ in the case or control group. ICD-10 diagnostic groups with an incidence of $<1 \%$ were combined in the main diagnosis groups. Only estimates for the ICD-10 diagnoses are reported from the second analysis, although the dummies for the main groups (including the remaining diagnosis) were included in the regression.

A patient could appear in more than one diagnosis group or have several ICD-10 diagnoses during the 3 years before or after diagnosis.

Not all patients had a full 3 year observation period before their diagnosis, so for the second and third years at the beginning of the period considered, patients' data were only available for 1 or 2 years, respectively. However, since this was also the case for the control group, we have included these shorter periods in the analysis.

The results are presented as ORs with 95\% CIs and associated p values. Extreme values were manually validated and no errors were identified. Cumulative incidence functions were estimated and are shown in figure 2. HRs were estimated using the Cox proportional hazard model. Two rates were estimated, the first in which no additional explanatory variables were included and, in the second, the explanatory variables were controlled for gender to determine whether there was any difference in mortality between males and females. The HR reflects the hazard of a patient dying compared with a control person dying.

\section{RESULTS}

We identified 2998 patients with OSA and 11974 control subjects. As expected, most of the patients with an initial diagnosis of OSA were middle-aged; one-quarter were women and approximately one-tenth were children or adolescents. The age distribution of the patients aged $0-19$ years is shown in figure 1 .

\section{Morbidity before and after a diagnosis of OSA}

Morbidities by percentage incidence and OR (with 95\% CI) are shown for the main diagnostic groups before a diagnosis of OSA and for diagnoses that occurred in $>1 \%$ of patients with OSA (table 1). OSA was positively associated with infections; endocrine, nutritional and metabolic diseases; nervous, eye and ENT disorders/respiratory system diseases (middle ear infections: OR $1.75,95 \%$ CI 1.21 to $2.52, \mathrm{p}<0.001$; tonsillitis: OR 1.74, 95\% CI 1.24 to $1.46, \mathrm{p}<0.01$; hypertrophic tonsils: OR $2.55,95 \%$ CI 1.74 to $3.72, \mathrm{p}<0.001$; asthmatic bronchitis: OR $1.34,95 \%$ CI 1.07 to $1.67, \mathrm{p}<0.01$ ); gastrointestinal diseases; skin conditions; congenital malformations, symptoms, signs and abnormal clinical or laboratory findings; and other factors that influence health status (suspected ENT disease: OR 
Figure 1 Diagnosis of obstructive sleep apnoea by age and gender.

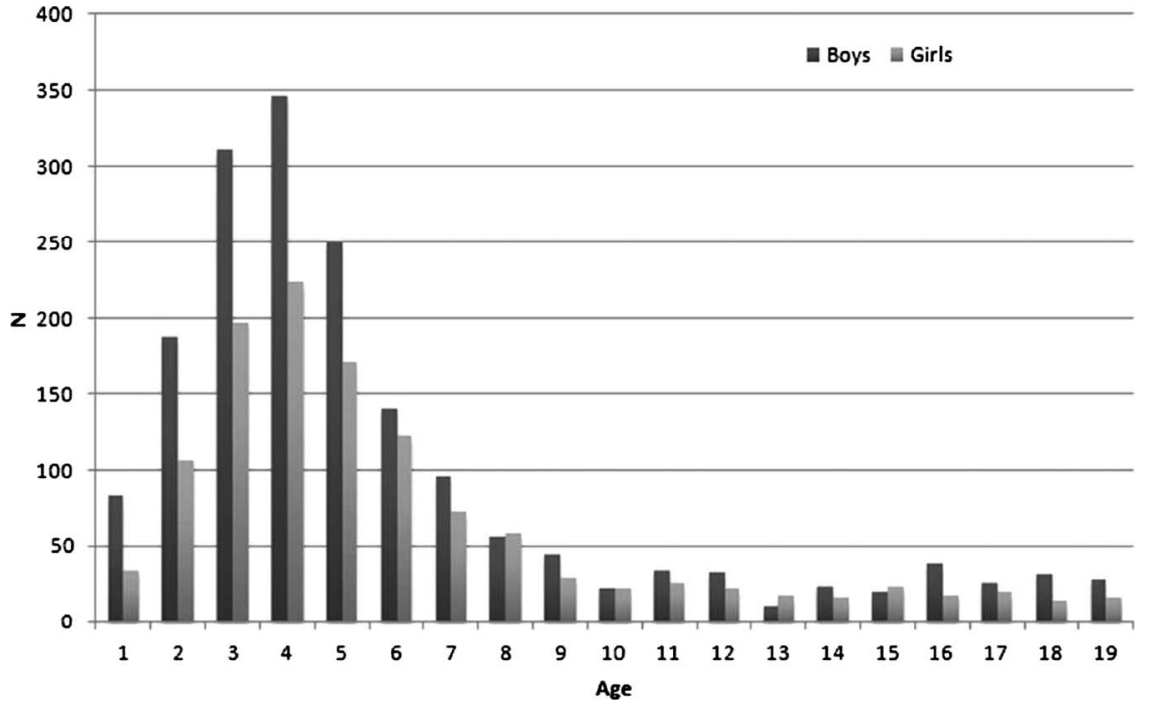

$1.80,95 \%$ CI 1.35 to $2.20, \mathrm{p}<0.001$; neurological disease: OR $1.17,95 \%$ CI 1.01 to $1.42, \mathrm{p}<0.01)$. Furthermore, although the overall frequency of injuries was not increased, there was a significant increase in the occurrence of head traumas (OR 1.49, 95\% CI 1.09 to $2.04, \mathrm{p}<0.01$ ) among children with OSA.

After a diagnosis of OSA (table 2) there were significantly higher incidences of the following conditions: endocrine, nutritional and metabolic diseases; nervous, eye and ENT disorders/ respiratory system diseases (middle ear infections: OR 1.78, 95\% CI 1.15 to $2.75, \mathrm{p}<0.001$; laryngitis: OR $1.81,95 \%$ CI 1.25 to 2.63, p <0.001; adenoid vegetations: OR 2.34, 95\% CI 1.57 to $3.51, \mathrm{p}<0.001$; hypertrophic tonsils: OR $2.46,95 \%$ CI 1.61 to $3.74, \mathrm{p}<0.001$; chronic respiratory insufficiency: OR 3.14 , $95 \%$ CI 1.72 to $5.74, \mathrm{p}<0.001$ ); skin and musculoskeletal conditions, congenital malformations, symptoms, signs and abnormal clinical or laboratory findings; and other factors that influence health status (suspected ENT disease: OR 1.71, 95\% CI 1.17 to 2.50, $\mathrm{p}<0.001$; neurological disease: OR 1.30, 95\% CI 1.05 to $1.62, \mathrm{p}<0.01)$.

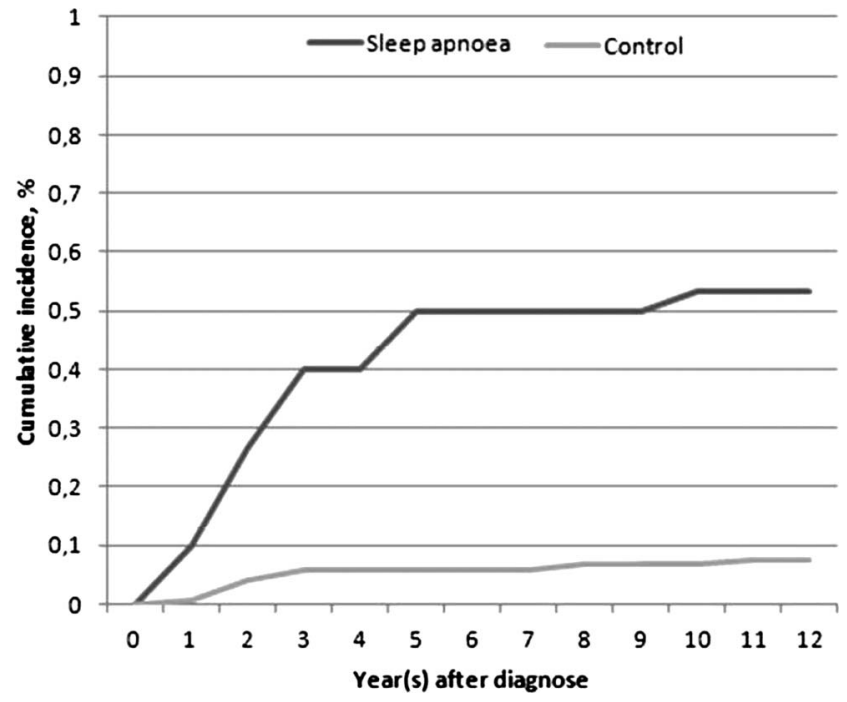

Figure 2 Mortality estimated as cumulative incidence in children with a diagnosis of sleep apnoea compared with controls.

\section{Mortality}

The 12-year death rates are shown in figure 2 and the number of individuals at risk are shown in table 3 . The 5 -year death rate was 70 per 10000 for patients and 11 per 10000 for controls. The HR for cases compared with controls was 6.58 (95\% CI 3.39 to $12.79, \mathrm{p}<0.001)$. Controlling for gender did not change the HR and the regression coefficient for gender was not statistically significant.

\section{DISCUSSION}

Our study shows that children diagnosed with OSA experienced a wide range of morbidities before their diagnosis. These included risk associations with upper airway abnormalities, upper airway infections, neurological, respiratory, gastrointestinal diseases and congenital malformations. After diagnosis the patients showed a greater incidence of a wide range of diseases including endocrine, nutritional and metabolic diseases; nervous, eye and ENT disorders; respiratory system diseases (upper airway problems and infections, asthma); and musculoskeletal problems. Mortality was higher in children with OSA than in controls. Our study did not consider whether this was related to the consequences of OSA or of comorbid diseases.

In adults, healthcare use and morbidity may be affected more than 8 years before a diagnosis of OSA. ${ }^{14}$ This period is inevitably shorter for younger children and is likely to be shorter for older children. Our study shows that premorbid conditions affect children at least 3 years before diagnosis. Although OSA is common in children, it is often underdiagnosed and therefore untreated. ${ }^{15}$ OSA is often first diagnosed when a child's function is affected, but specific data regarding the extent, severity and consequences are sparse. OSA in patients of normal weight with adenotonsils/hypertrophic tonsils is common and they often recover after appropriate surgery, with improvements in symptoms, better daytime and cognitive function, certain biomarkers, lower incidence of cardiac abnormalities and reduced healthcare use. ${ }^{16} 17$ This is much less often the case for overweight children, however. ${ }^{18}$ Relatively little is known about the effect of treatment with continuous positive airway pressure (CPAP) in children, the information we have being based on case series. ${ }^{19-21}$ The evaluation of the effect of CPAP in children is limited by its use in patients with special needs such as cerebral palsy or other neurological, muscular and genetic disorders. ${ }^{22-24}$ 
Table 1 Morbidities 3 years before a diagnosis of sleep apnoea by major disease groups

\begin{tabular}{|c|c|c|c|c|c|c|}
\hline \multirow[b]{4}{*}{ Disease group } & \multicolumn{6}{|c|}{ Obstructive sleep apnoea } \\
\hline & \multirow[b]{3}{*}{ Cases N (\%) } & \multirow[b]{3}{*}{ Control N (\%) } & \multicolumn{4}{|l|}{$\mathrm{OR}^{*}$} \\
\hline & & & \multirow[b]{2}{*}{ Estimate } & \multicolumn{2}{|c|}{$95 \% \mathrm{Cl}$} & \multirow[b]{2}{*}{ p Value } \\
\hline & & & & Lower & Upper & \\
\hline Infectious and parasitic diseases & $224(8.1)$ & $1274(4.0)$ & 1.19 & 1.01 & 1.40 & 0.0339 \\
\hline Neoplasms & $25(0.9)$ & $35(0.3)$ & 1.66 & 1.05 & 2.63 & 0.0299 \\
\hline Blood and immunological diseases & $19(0.7)$ & $28(0.3)$ & 1.20 & 0.71 & 2.03 & 0.4899 \\
\hline Endocrine, nutritional and metabolic diseases & $54(1.9)$ & $83(0.7)$ & 1.30 & 0.94 & 1.80 & 0.1134 \\
\hline Mental and psychiatric disorders & $21(0.8)$ & $47(0.4)$ & 0.91 & 0.54 & 1.53 & 0.7157 \\
\hline Nervous system disorders & $112(4.0)$ & $72(0.6)$ & 2.12 & 1.65 & 2.73 & $<0.0001$ \\
\hline Diseases of the eye and adnexa & $69(2.5)$ & $88(0.8)$ & 1.43 & 1.07 & 1.90 & 0.0148 \\
\hline Ear, nose and throat diseases & $159(5.7)$ & $220(2.0)$ & 1.61 & 1.33 & 1.94 & $<0.0001$ \\
\hline Circulatory/cardiovascular diseases & $17(0.6)$ & $16(0.1)$ & 1.41 & 0.81 & 2.45 & 0.2229 \\
\hline Respiratory diseases & $581(21.0)$ & $1068(9.6)$ & 1.78 & 1.60 & 1.98 & $<0.0001$ \\
\hline Gastrointestinal diseases & $117(4.2)$ & $249(2.2)$ & 1.34 & 1.09 & 1.66 & 0.0064 \\
\hline Skin and subcutaneous tissue diseases & $77(2.8)$ & $153(1.4)$ & 1.32 & 1.02 & 1.71 & 0.0374 \\
\hline Musculoskeletal system and connective tissue diseases & $94(3.4)$ & $230(2.1)$ & 1.23 & 0.98 & 1.56 & 0.0789 \\
\hline Genitourinary diseases & $58(2.1)$ & $130(1.2)$ & 1.15 & 0.85 & 1.54 & 0.3633 \\
\hline Pregnancy, childbirth and puerperium & $1(0.0)$ & $3(0.0)$ & - & - & - & 0.8266 \\
\hline Certain conditions originating in the perinatal period & $55(2.0)$ & $97(0.9)$ & 1.27 & 0.94 & 1.71 & 0.1242 \\
\hline Congenital malformations, deformations and chromosomal abnormalities & $201(7.3)$ & $315(2.8)$ & 1.56 & 1.31 & 1.85 & $<0.0001$ \\
\hline Abnormal clinical and laboratory findings & $318(11.5)$ & $685(6.2)$ & 1.21 & 1.06 & 1.39 & 0.0061 \\
\hline Injury, poisoning and certain other external causes & $881(31.8)$ & $2102(27.9)$ & 1.09 & 0.99 & 1.19 & 0.0686 \\
\hline External causes of morbidity and mortality & $0(0.0)$ & $1(0.0)$ & - & - & - & - \\
\hline Other factors influencing health status and contact with health services & $698(25.2)$ & $2771(15.1)$ & 1.29 & 1.16 & 1.43 & $<0.0001$ \\
\hline
\end{tabular}

${ }^{*}$ Conditional logit, baseline: no morbidity.

Table 2 Morbidities 3 years after a diagnosis of sleep apnoea by major disease groups

\begin{tabular}{|c|c|c|c|c|c|c|}
\hline \multirow[b]{4}{*}{ Disease group } & \multicolumn{6}{|c|}{ Obstructive sleep apnoea } \\
\hline & \multirow[b]{3}{*}{ Cases $\mathrm{N}(\%)$} & \multirow[b]{3}{*}{ Control N (\%) } & \multicolumn{4}{|l|}{$\mathrm{OR}^{*}$} \\
\hline & & & \multirow[b]{2}{*}{ Estimate } & \multicolumn{2}{|l|}{$95 \% \mathrm{Cl}$} & \multirow[b]{2}{*}{$\mathrm{p}$ Value } \\
\hline & & & & Lower & Upper & \\
\hline Infectious and parasitic diseases & $78(3.0)$ & $158(1.5)$ & 1.00 & 0.76 & 1.31 & 0.9764 \\
\hline Neoplasms & $24(0.9)$ & $48(0.5)$ & 0.98 & 0.60 & 1.60 & 0.9356 \\
\hline Blood and immunological diseases & $9(0.3)$ & $29(0.3)$ & 0.85 & 0.41 & 1.78 & 0.6704 \\
\hline Endocrine, nutritional and metabolic diseases & $63(2.4)$ & $65(0.6)$ & 1.78 & 1.29 & 2.45 & 0.0004 \\
\hline Mental and psychiatric disorders & $49(1.9)$ & $104(1.0)$ & 1.22 & 0.88 & 1.70 & 0.2268 \\
\hline Nervous system disorders & $185(7.2)$ & $81(0.8)$ & 3.16 & 2.58 & 3.89 & 0.0000 \\
\hline Diseases of the eye and adnexa & $65(2.5)$ & $81(1.1)$ & 1.09 & 0.81 & 1.46 & 0.5669 \\
\hline Ear, nose and throat diseases & $101(3.9)$ & $143(1.4)$ & 1.45 & 1.14 & 1.84 & 0.0022 \\
\hline Circulatory/cardiovascular diseases & $17(0.7)$ & $23(0.2)$ & 1.09 & 0.58 & 2.05 & 0.7818 \\
\hline Respiratory diseases & $363(14.1)$ & $510(5.0)$ & 1.94 & 1.70 & 2.22 & $<0.0001$ \\
\hline Gastrointestinal diseases & $113(4.4)$ & $243(2.4)$ & 1.14 & 0.91 & 1.43 & 0.2563 \\
\hline Skin and subcutaneous tissue diseases & $63(2.4)$ & $114(1.1)$ & 1.42 & 1.06 & 1.89 & 0.0193 \\
\hline Musculoskeletal system and connective tissue diseases & $93(3.6)$ & $217(2.1)$ & 1.29 & 1.01 & 1.64 & 0.0390 \\
\hline Genitourinary diseases & $53(2.1)$ & $195(1.9)$ & 0.92 & 0.68 & 1.25 & 0.6005 \\
\hline Pregnancy, childbirth and puerperium & $8(0.3)$ & $31(0.3)$ & 0.73 & 0.30 & 1.75 & 0.4751 \\
\hline Certain conditions originating in the perinatal period & $23(0.9)$ & $15(0.1)$ & 2.24 & 1.30 & 3.84 & 0.0036 \\
\hline Congenital malformations, deformations and chromosomal abnormalities & $172(6.7)$ & $209(2.0)$ & 1.83 & 1.51 & 2.22 & $<0.0001$ \\
\hline Abnormal clinical and laboratory findings & $252(9.8)$ & $452(4.4)$ & 1.37 & 1.17 & 1.60 & 0.0001 \\
\hline Injury, poisoning and certain other external causes & $874(34.0)$ & $2971(28.9)$ & 1.16 & 1.06 & 1.27 & 0.0018 \\
\hline External causes of morbidity and mortality & $0(0.0)$ & $3(0.0)$ & 0.00 & - & - & - \\
\hline Other factors influencing health status and contact with health services & $562(21.9)$ & $1274(12.4)$ & 1.35 & 1.20 & 1.51 & $<0.0001$ \\
\hline
\end{tabular}

${ }^{*}$ Conditional logit, baseline: no morbidity. 
Table 3 Number of individuals at risk

\begin{tabular}{|c|c|c|c|c|c|c|c|}
\hline & \multicolumn{7}{|l|}{ Year } \\
\hline & 0 & 2 & 4 & 6 & 8 & 10 & 12 \\
\hline $\begin{array}{l}\text { Sleep } \\
\text { apnoea }\end{array}$ & 2998 & 2988 & 2977 & 2972 & 2971 & 2971 & 2962 \\
\hline Control & 11974 & 11968 & 11961 & 11962 & 11962 & 11962 & 11942 \\
\hline
\end{tabular}

The current study of children and adolescents up to 19 years of age was done to evaluate potential known and unidentified comorbidities in OSA, and showed that many known risks are associated with a later diagnosis of OSA (eg, upper airway abnormalities, asthma and neurological problems). However, other formerly unrecognised diseases seem to be more closely associated with a later diagnosis of OSA in children (eg, selected infections, gastrointestinal, skin and musculoskeletal diseases, and clinical evaluation for a number of health-related problems). This implies that OSA not only exists in children evaluated for adenotonsillectomy. It is likely that certain health-related problems coexist or are associated with one another (eg, obesity, congenital malformations, neurological disease and diabetes), but our study shows that children and teenagers with OSA have more contact with the healthcare system for a wide range of health-related issues before and after their OSA diagnosis, which supports the findings of former studies. ${ }^{25}$ This could in part be due to obesity in these patients, probably due to underregistration of overweight in children. It has been claimed that attention deficit hyperactivity disorder is associated with OSA in case series, although carefully controlled trials have failed to find evidence of this. ${ }^{26}$ This is underlined by our finding that there was no greater incidence of psychiatric disease either before or after an OSA diagnosis.

There are a number of limitations to this study. First, it is based on clinical and hospital reports supplied to the NPR. The diagnostic accuracy depends on the institution's presentation and reporting of the diagnosis and the comorbidities. Confounder variables (eg, BMI) were not recorded, and symptoms and the results of clinical evaluations (eg, PSG and cardiac evaluations) were not considered. PSG is not performed in all subjects as there is tendency to overdiagnose OSA in the childhood population. For these reasons, we cannot relate the findings to OSA. Second, we excluded sleep disordered breathing among controls by diagnosis but not by nocturnal measures; OSA/heavy snoring would be expected in $0.7-4.3 \%$ of children. ${ }^{27}$ Analysing subgrouped diseases incurs a risk of type 1 error. However, the strength of the study is that it considers data from a national database that includes all identified patients that is time-locked (all reports must be associated with the patient contacts) and covers a substantial follow-up period. Obesity and probably OSA often tend to occur disproportionately in lower socioeconomic groups. We adjusted for SES in our selection of the controls. Furthermore, we randomly chose controls without a diagnosis of sleep disordered breathing (snoring, sleep apnoea, hypoventilation), although they may suffer from other diseases. As a consequence of these factors, our method is conservative and the real differences between the groups are likely to be larger than reported here.

To ameliorate the consequences of OSA, a number of issues should be considered: increased diagnostic capacity; improved treatment and management facilities; the identification of highrisk groups for screening procedures; and general interventions for high-risk groups (eg, obesity) in children. Furthermore, despite the limited effect of adenotonsillectomy, there is only limited postoperative screening of high-risk groups. There is a need to reinforce our knowledge of the long-term effects of OSA in children and to evaluate the effects of interventions, including those on secondary health problems. Consequently, a better diagnostic capacity and treatment facilities for the paediatric population are required. The prediagnostic probability may be improved using information gleaned from questionnaires, although this also carries a risk of overlooking high-risk individuals. The current study suggests that OSA is more likely to occur with certain disorders (eg, upper airway infections and structural abnormalities, and respiratory/neurological diseases) in which OSA should be considered. OSA in obese children should also be taken into account. A number of children with a diagnosis of OSA have undergone adenotonsillectomy. In the future, evaluation of the population should include a subgroup analysis of treatment interventions.

In summary, our study shows that children and teenagers have a wide range of morbidities at least 3 years before and after a diagnosis of OSA, and that mortality is higher in paediatric OSA than in controls.

Contributors PJ and JK created, initiated and managed the project. PJ is the main author. JK and RI performed the statistical analyses, supervised by PJ.

Funding This study was supported by an unrestricted grant from the Philips Respironics Foundation and the Center for Healthy Aging, Faculty of Health Sciences, University of Copenhagen.

\section{Competing interests None.}

Ethics approval The study was approved by the Danish Data Protection Agency. As the data handled were anonymised, neither individual nor ethical approval was required.

Provenance and peer review Not commissioned; externally peer reviewed.

Data sharing statement The data contain an extensive dataset from all national data. The current data are created as a separate data file located in Denmark's Civil Registration System Statistics. There are further data containing all information about the diagnoses, social factors, mortalities and parenteral information.

\section{REFERENCES}

1 Carno MA, Ellis E, Anson E, et al. Symptoms of sleep apnea and polysomnography as predictors of poor quality of life in overweight children and adolescents. $J$ Pediatr Psychol 2008;33:269-78.

2 Mitchell RB, Kelly J. Outcome of adenotonsillectomy for obstructive sleep apnea in obese and normal-weight children. Otolaryngol Head Neck Surg 2007;137:43-8.

3 Di Francesco R, Monteiro R, Paulo ML, et al. Craniofacial morphology and sleep apnea in children with obstructed upper airways: differences between genders. Sleep Med 2012;13:616-20.

4 Seddon PC, Khan Y. Respiratory problems in children with neurological impairment. Arch Dis Child 2003;88:75-8

5 Archbold KH, Vasquez MM, Goodwin JL, et al. Effects of sleep patterns and obesity on increases in blood pressure in a 5-year period: report from the Tucson children's assessment of sleep apnea study. J Pediatr 2012;161:26-30.

6 O'Brien LM, Lucas NH, Felt BT, et al. Aggressive behavior, bullying, snoring, and sleepiness in schoolchildren. Sleep Med 2011;12:652-8.

7 Gozal D, Kheirandish-Gozal L. Obesity and excessive daytime sleepiness in prepubertal children with obstructive sleep apnea. Pediatrics 2009;123:13-18.

8 Landau YE, Bar-Yishay 0, Greenberg-Dotan S, et al. Impaired behavioral and neurocognitive function in preschool children with obstructive sleep apnea. Pediatr Pulmonol 2012;47:180-8.

9 Bourke R, Anderson V, Yang JS, et al. Cognitive and academic functions are impaired in children with all severities of sleep-disordered breathing. Sleep Med 2011;12:489-96.

10 Rudnick EF, Mitchell RB. Behavior and obstructive sleep apnea in children: is obesity a factor? Laryngoscope 2007;117:1463-6.

11 Mulvaney SA, Goodwin JL, Morgan WJ, et al. Behavior problems associated with sleep disordered breathing in school-aged children: the Tucson children's assessment of sleep apnea study. J Pediatr Psychol 2006;31:322-30.

12 Rosen CL, Palermo TM, Larkin EK, et al. Health-related quality of life and sleep-disordered breathing in children. Sleep 2002:25:657-66. 
13 Perkin RM, Young T. Obstructive sleep apnea in children. Reversing the trend of underdiagnosis. Adv Nurse Pract 2000;8:57-9.

14 Jennum P, Kjellberg J. Health, social and economical consequences of sleep-disordered breathing: a controlled national study. Thorax 2011;66:560-6.

15 Pijpers M, Poels PJ, Vaandrager JM, et al. Undiagnosed obstructive sleep apnea syndrome in children with syndromal craniofacial synostosis. J Craniofac Surg 2004;15:670-4.

16 Bonuck KA, Freeman K, Henderson J. Growth and growth biomarker changes after adenotonsillectomy: systematic review and meta-analysis. Arch Dis Child 2009;94:83-91.

17 Tarasiuk A, Simon T, Tal A, et al. Adenotonsillectomy in children with obstructive sleep apnea syndrome reduces health care utilization. Pediatrics 2004;113:351-6.

18 Costa DJ, Mitchell R. Adenotonsillectomy for obstructive sleep apnea in obese children: a meta-analysis. Otolaryngol Head Neck Surg 2009;140:455-60.

19 Waters KA, Everett FM, Bruderer JW, et al. Obstructive sleep apnea: the use of nasal CPAP in 80 children. Am J Respir Crit Care Med 1995;152:780-5.

20 Marcus CL, Radcliffe J, Konstantinopoulou S, et al. Effects of positive airway pressure therapy on neurobehavioral outcomes in children with obstructive sleep apnea. Am J Respir Crit Care Med 2012;185:998-1003.
21 Castorena-Maldonado A, Torre-Bouscoulet L, Meza-Vargas S, et al. Preoperative continuous positive airway pressure compliance in children with obstructive sleep apnea syndrome: assessed by a simplified approach. Int J Pediatr Otorhinolaryngol 2008;72:1795-800

22 Hsiao KH, Nixon GM. The effect of treatment of obstructive sleep apnea on quality of life in children with cerebral palsy. Res Dev Disabil 2008;29:133-40.

23 Levanon A, Tarasiuk A, Tal A. Sleep characteristics in children with Down syndrome. J Pediatr 1999;134:755-60.

24 Tarasiuk A, Abdul-Hai A, Moser A, et al. Sleep disruption and objective sleepiness in children with beta-thalassemia and congenital dyserythropoietic anemia. Arch Pediatr Adolesc Med 2003;157:463-8.

25 Tarasiuk A, Greenberg-Dotan S, Simon-Tuval T, et al. Elevated morbidity and health care use in children with obstructive sleep apnea syndrome. Am J Respir Crit Care Med 2007;175:55-61.

26 Galland BC, Tripp EG, Gray A, et al. Apnea-hypopnea indices and snoring in children diagnosed with ADHD: a matched case-control study. Sleep Breath 2011;15:455-62.

27 Stone RS, Spiegel JH. Prevalence of obstructive sleep disturbance in children with failure to thrive. J Otolaryngol Head Neck Surg 2009;38:573-9. 